Proceedings of the Edinburgh Mathematical Society (2003) 46, 451-464 (C)

DOI:10.1017/S0013091501000827 Printed in the United Kingdom

\title{
BOUNDARY-CHAOTIC BEHAVIOUR OF CONTINUOUS FUNCTIONS UNDER THE ACTION OF OPERATORS
}

\author{
LUIS BERNAL-GONZÁLEZ AND MARÍA DEL CARMEN CALDERÓN-MORENO \\ Departamento de Análisis Matemático, Facultad de Matemáticas, \\ Universidad de Sevilla, Apdo. 1160, Avenida Reina Mercedes, \\ 41080 Sevilla, Spain (lbernal@us.es; mccm@us.es)
}

(Received 11 September 2001)

\begin{abstract}
In this paper we introduce two classes of operators on spaces of continuous functions with values in $F$-spaces under the action of which many functions behave chaotically near the boundary. Several examples - including onto linear operators, left and right composition operators, multiplication operators, and operators with pointwise dense range or with some stability property - are given. This new theory extends one recently developed on spaces of holomorphic functions.
\end{abstract}

Keywords: omnipresent operator; DI-operator; spaces of continuous functions; cluster set; boundary-chaotic function; composition operator

2000 Mathematics subject classification: Primary 47B38

Secondary 30D40; 46E10; 54D45

\section{Introduction}

In this paper we are concerned with the chaotic behaviour near the boundary exhibited by certain continuous functions under the action of several kinds of operator. Such chaotic behaviour has attracted the attention of many mathematicians in the past few decades, mainly in the setting of holomorphic or meromorphic functions on complex domains. Most results obtained in this field are directly or indirectly related to cluster sets. We refer the reader to $[\mathbf{8}]$ and $[\mathbf{1 9}]$ for definitive surveys about the matter.

Let us introduce the following rather general definition of cluster set. Assume that $X$, $Y$ are topological spaces and that $G$ is an open subset of $X$ with non-empty boundary $\partial G$. Let $f: G \rightarrow Y$ be a mapping. If $t \in \partial G$, then the cluster set of $f$ at $t$ is defined as the set

$$
S(f, t)=\bigcap\{\overline{f(V \cap G)}: V \subset X, V \ni t, V \text { open }\},
$$

where $\bar{A}$ denotes the closure of a subset $A$. Observe that if $t$ has a denumerable basis of neighbourhoods, then $S(f, t)$ is the set of all $y \in Y$ for which there exists a sequence of points $\left(z_{n}\right) \subset G$ with $z_{n} \rightarrow t$ and $f\left(z_{n}\right) \rightarrow y$ as $n \rightarrow \infty$. We are interested in the existence of boundary-chaotic functions $f$ in the following sense. 
A function $f: G \rightarrow Y$ is said to be boundary chaotic if and only if each cluster set $S(f, t)$ is maximal, i.e.

$$
S(f, t)=Y \quad \text { for all } t \in \partial G .
$$

For instance, in the case $X=Y=\mathbb{C}$, the complex plane, if $f$ is holomorphic in a punctured neighbourhood of $t$ and $t$ is an essential singularity of $f$, then $S(f, t)$ is maximal. The continuous function $f(x)=x^{-1} \sin \left(x^{-1}\right)$ is a trivial example for $X=Y=$ $\mathbb{R}$, the real line and $G=(0,+\infty)$.

If $G$ is a non-empty open subset of $\mathbb{C}$, then we denote the Fréchet space (hence a Baire space) of holomorphic functions in $G$, endowed with the compact-open topology, as usual, by $H(G)$. Through the introduction of the 'omnipresent operators' on $H(G)$, The first author showed that most functions (in the sense of Baire) in $H(G)$ together with all their derivatives and antiderivatives are boundary chaotic (see $[\mathbf{1}]$ ). This can also be extracted (with different methods) from the results of Grosse-Erdmann [10, Chapter 3] (see also $[\mathbf{1 1}, \S 4 \mathrm{~b}]$ ), who in turn improves some statements of Luh about the existence of 'holomorphic monsters' (see [14]). See also [15], [16] and [20] for further development of these topics. In $[\mathbf{1}]$ the omnipresence is also shown for a rather general class of integral operators.

The theory created by Luh and Grosse-Erdmann was recently extended by the authors in $[\mathbf{3}]$ via the introduction of the ' $T$-monsters' and of the 'strongly omnipresent operators' (these are a special case of omnipresent operators). Several examples of this kind of operator, including those of the form $\Phi(D), \Phi\left(D^{-1}\right)$ (where $D$ denotes differentiation and $\Phi$ denotes an adequate non-zero holomorphic function), are furnished in $[\mathbf{3}]$. Additional examples can be found in [5] and [6]. The strongly omnipresent operators are related to certain generalized cluster sets introduced by Luh [14], who used affine linear transformations $z \mapsto a z+b$ for his definition; hence, unlike the omnipresent operators, a natural extension to general topological spaces does not seem to be possible for the strong omnipresence.

On the other hand, the first author proved in $1995[\mathbf{2}]$ that, given any non-relatively compact subset $A \subset G$, most functions $f$ in $H(G)$ have the property that $f^{(n)}(A)$ is dense in $\mathbb{C}$ for every $n=0,1,2, \ldots$ The second author has recently established that a wide class of differential operators $\Phi(D)$ and of integral operators shares the same property [7]. This has led us to introduce the concept of 'dense-image operators' or, in short, 'DI-operators' (see [4]). It happens that an operator on $H(G)$ is omnipresent whenever it is a DI-operator. This concept can also be defined on more general topological spaces (see $\S 2$ ).

Our aim in this paper is to study the omnipresent operators and the DI-operators on spaces of continuous functions, as well as to provide several (general or more concrete) examples (see $\S \S 2-4$ ). We want to point out that while Runge-Mergelyan's theorems are the natural basic tools to attack the corresponding problems on spaces of holomorphic functions, we cannot use them, of course, in the setting of continuous functions. In addition, observe that in the case $Y=\mathbb{C}, G=$ an open subset of $\mathbb{C}$, we have that the space $H(G)$ is closed (hence non-dense) in $C(G, Y):=\{$ continuous functions $f: G \rightarrow Y$ \}. Consequently, even though an operator $T$ on $C(G, Y)$ may take $H(G)$ into itself, we cannot 
guarantee from the omnipresence (or from the property DI) of $T_{\left.\right|_{H(G)}}$ that $T$ itself has the same property. This justifies an independent study into the class of continuous functions.

\section{Definitions and preliminary results}

From now on, $X$ will denote a Hausdorff, second-countable, locally compact topological space, $G$ will be an open $\sigma$-compact subset of $X$ with $\partial G \neq \emptyset$, and $Y$ will stand for a separable $F$-space (i.e. a metrizable complete topological vector space). Observe that if $\partial G \neq \emptyset$, then $\emptyset \neq G \neq X$. Conversely, under the additional hypothesis of connectedness of $X$, the latter condition guarantees that the boundary of $G$ is non-empty. Indeed, if $\partial G=\emptyset$, then $\bar{G} \backslash G=\bar{G} \backslash G^{0}=\emptyset$, where $A^{0}$ denotes the interior of a subset $A$. Therefore, $G=\bar{G}$, so $G$ is open and closed, which contradicts the connectedness of $X$. We will promptly need $G$ to be Hausdorff, locally compact and $\sigma$-compact. The first two properties are inherited from $X$ (the second one due to the fact that $G$ is open), but not the third one. This is why we have to impose that $G$ be $\sigma$-compact. Indeed, if $A$ is any non-denumerable set with the discrete topology, then $A$ is, trivially, Hausdorff, locally compact and non-compact, and if $X=A \cup\{w\}$ is its Alexandroff compactification (see, for example, [18]), then $X$ is $\sigma$-compact (since it is compact), Hausdorff and locally compact, $A$ is open in $X, \partial A \neq \emptyset$ (because $\partial A=\{w\}$ ), but $A$ is not $\sigma$-compact due to non-denumerability.

Since our $G$ is $\sigma$-compact, there is a sequence $\left(K_{n}\right)$ of compact subsets with $K_{n} \subset K_{n+1}^{0}$ for all $n$ and $G=\bigcup_{n=1}^{\infty} K_{n}$ (see, for example, [12, pp. 325, 326]). From this it is easy to see that if $K$ is a compact subset of $G$, then $K \subset K_{n}$ for some $n$. Therefore, the same construction given in $[\mathbf{9}$, Chapter 7$]$ and $[\mathbf{1 3}$, p. 136] can be carried over in order to make the linear space $C(G, Y)$ an $F$-space (hence a Baire space) with, for instance, the distance

$$
\rho(f, g)=\sum_{n=1}^{\infty} \frac{1}{2^{n}} \frac{\max _{x \in K_{n}} d(f(x), g(x))}{1+\max _{x \in K_{n}} d(f(x), g(x))},
$$

where $d$ is any complete translation-invariant distance on $Y$. The $\rho$-convergence is precisely the uniform convergence on compact subsets of $G$. It is well known that the family of sets

$$
D(g, K, \varepsilon)=\{f \in C(G, Y): d(f(x), g(x))<\varepsilon \forall z \in K\}
$$

where $\varepsilon>0, g \in C(G, Y)$ and $K \subset G$ is compact, is a basis for the topology given on $C(G, Y)$. An operator on $C(G, Y)$ always refers to a continuous (not necessarily linear) self-mapping $T: C(G, Y) \rightarrow C(G, Y)$.

Denote $O(\partial G):=\{V \subset X: V$ is open and $V \cap \partial G \neq \emptyset\}$. Given subsets $A \subset X, B \subset Y$ and an operator $T$ on $C(G, Y)$, let us denote

$$
R(T, A, B)=\{f \in C(G, Y): \text { there exists } a \in A \cap G \text { with } T f(a) \in B\} .
$$


Remark 2.1.

(a) It is evident that $R(T, A, B) \subset R\left(T, A^{\prime}, B^{\prime}\right)$ if $A \subset A^{\prime}$ and $B \subset B^{\prime}$.

(b) The set $R(T, A, B)$ is open for all $A \subset X$ whenever $B$ is open. Indeed, we have that

$$
R(T, A, B)=\bigcup\left\{\varphi_{a}^{-1}(B): a \in A \cap G\right\},
$$

where $\varphi_{a}$ is the evaluation mapping $\varphi_{a}: f \in C(G, Y) \mapsto T f(a) \in Y$, which is clearly continuous.

For an operator $T$ on $C(G, Y)$ we introduce the followings definitions.

We say that $T$ is omnipresent if and only if $R(T, V, W)$ is dense in $C(G, Y)$ for every $V \in O(\partial G)$ and every non-empty open subset $W \subset Y$.

We say that $T$ is a DI-operator if and only if $R(T, A, W)$ is dense in $C(G, Y)$ for every non-relatively compact subset $A$ in $G$ and every non-empty open subset $W \subset Y$.

Note that each DI-operator is omnipresent, because $V \cap G$ is non-relatively compact in $G$ for every $V \in O(\partial G)$. Indeed, pick $t \in V \cap \partial G$ and assume, by way of contradiction, that there is a compact set $K$ with $V \cap G \subset K \subset G$. Then $(X \backslash K) \cap V$ is an open subset of $X$ containing $t$, hence $G \cap(X \backslash K) \cap V \neq \emptyset$, so $V \cap G \not \subset K$, which is absurd. In $\S 4$ several examples of omnipresent operators which are not DI-operators can be found.

Denote by $C h(T)$ the set of functions $f \in C(G, Y)$ such that $T f$ is boundary-chaotic (see $\S 1$ ). If $A \subset G$, we denote by $M(T, A)$ the set

$$
M(T, A)=\{f \in C(G, Y): T f(A) \text { is dense in } Y\} .
$$

It should be noted that

$$
C h(T)=\bigcap R(T, V, W)
$$

and

$$
M(T, A)=\bigcap R(T, A, W)
$$

where $V$ runs over the members of $O(\partial G)$ and $W$ runs over all non-empty open subsets of $Y$. In particular, $C h(T)$ and $M(T, A)$ are $G_{\delta}$ subsets of $C(G, Y)$. Observe that if $A$ is relatively compact, then $g(A)$ is dense in $Y$ for no continuous function $g$. The following proposition shows that under adequate assumptions on $X$ and $Y$, the fact ' $T$ is omnipresent' (or ' $T$ is a DI-operator') means, roughly speaking, that 'most functions behave wildly near the boundary under the action of $T$ '. Recall that, in a Baire space, a subset is residual if and only if its complement is of first category.

Proposition 2.2. Assume that $T$ is an operator on $C(G, Y)$. We have the following.

(a) The operator $T$ is a DI-operator if and only if $M(T, A)$ is dense for every nonrelatively compact subset $A \subset G$ if and only if $M(T, A)$ is residual for every nonrelatively compact subset $A \subset G$.

(b) The operator $T$ is omnipresent if and only if $C h(T)$ is dense if and only if $C h(T)$ is residual. 
Proof. Since $Y$ is separable we can fix a denumerable open basis $\left(W_{n}\right)$ for $Y$. Since $X$ is also second-countable, we can also fix a denumerable open basis for $X$ and extract from it the sequence $\left(V_{j}\right)$ of members meeting $\partial G$. Then (a) and (b) follow at once from Remark 2.1, from the fact that $C(G, Y)$ is a Baire space, and from the equalities (derived from $(2.2),(2.3))$ :

$$
M(T, A)=\bigcap_{n \in \mathbb{N}} R\left(T, A, W_{n}\right), \quad C h(T)=\bigcap_{j, n \in \mathbb{N}} R\left(T, V_{j}, W_{n}\right) .
$$

The next auxiliary extension result will prove very useful for discovering chaotic behaviour.

Lemma 2.3. Assume that $x_{0} \in G, y_{0} \in Y$ and that $K$ is a compact subset of $G$ with $x_{0} \notin K$. If $g \in C(G, Y)$, then there exists $h \in C(G, Y)$ such that $h\left(x_{0}\right)=y_{0}$ and $h(x)=g(x)$ for all $x \in K$.

Proof. Since $G$ is Hausdorff, $K$ is closed. Since $G$ is Hausdorff and locally compact, it is a Tychonoff space [17], whence there exists a continuous function $f: G \rightarrow \mathbb{K}$ such that $f\left(x_{0}\right)=0$ and $f(x)=1$ for all $x \in K$. Here $\mathbb{K}=\mathbb{R}$ or $\mathbb{C}$ is the base field of $Y$. Since $Y$ is a topological vector space, the mapping $h: G \rightarrow Y$ given by

$$
h(x)=f(x) g(x)+(1-f(x)) y_{0}
$$

is continuous. A simple glance shows that $h$ satisfies the required properties.

A more direct proof of the last lemma (using distances to construct the auxiliary function $f$ ) can be made by taking into account that $X$ (hence $G$ also) is metrizable, since any Hausdorff locally compact second-countable space is metrizable [12, p. 342]. Nevertheless, we will not use this property.

To finish this section, we propose the following definitions in order to isolate several conditions which will make an appearance during the next section. Assume that $T$ is an operator on $C(G, Y)$.

We say that $T$ is pointwise stable near the boundary if and only if for every compact subset $K \subset G$ there exists a compact subset $M \subset G$ with the property that for every $a \in G \backslash M$, every $f \in C(G, Y)$ and every neighbourhood $W$ of $T f(a)$ there exists a point $b \in G \backslash K$ such that if $g \in C(G, Y)$ and $g(b)=f(b)$, then $T g(a) \in W$.

The property $T g(a) \in W$ can be expressed, of course, in terms of the distance in $Y$. The second concept is as follows.

We say that $T$ is somewhere pointwise stable near the boundary if and only if for every compact subset $K \subset G$ and every $V \in O(\partial G)$ there exists a point $a \in V \cap G$ with the property that for every $f \in C(G, Y)$ and every neighbourhood $W$ of $T f(a)$ there exists $a$ point $b \in G \backslash K$ such that if $g \in C(G, Y)$ and $f(b)=g(b)$, then $T g(a) \in W$. 
As an example, the reflection $T f(x)=f(-x)$ (where $X:=\mathbb{R}, x \in G:=(-1,1)$ ) is pointwise stable near the boundary. We will give other examples in $\S 4$. It is easy to see that if an operator $T$ is pointwise stable near the boundary, then it is somewhere pointwise stable near the boundary. Finally, a pair of definitions related to density are introduced.

We say that $T$ has pointwise dense range near the boundary whenever there is a compact subset $K \subset G$ such that the set $\{T f(a): f \in C(G, Y)\}$ is dense in $Y$ for every $a \in G \backslash K$.

A corresponding weaker property is the following.

We say that $T$ has somewhere pointwise dense range near the boundary if and only if for each $V \in O(\partial G)$ there exists a point $a \in V \cap G$ such that $\{T f(a): f \in C(G, Y)\}$ is dense in $Y$.

Trivially, dense range implies pointwise dense range.

\section{General theory and some examples}

Our objectives are to produce DI-operators and omnipresent operators from other such operators as well as to furnish sufficient conditions for an operator to be DI or omnipresent. We also want to provide concrete examples of these kinds of operator. Therefore, it is natural to wonder whether an easy example is available to start with. Surprisingly, the easiest operator does the job.

Theorem 3.1. The identity operator on $C(G, Y)$ is a DI-operator. Hence it is omnipresent.

Proof. Fix a non-relatively compact subset $A$ in $G$ and a non-empty open subset $W \subset Y$. Consider the operator $T$ given by $T f=f$ and a basic neighbourhood $D(g, K, \varepsilon)$ like that in (2.1). Since $R(T, A, W)=\{f \in C(G, Y): \exists a \in A$ with $f(a) \in W\}$, we have to find a function $h \in C(G, Y)$ and a point $a \in A$ in such a way that $d(h(x), g(x))<$ $\varepsilon \forall z \in K$ and $h(a) \in W$. Since $A$ is non-relatively compact, there is $a \in(G \backslash K) \cap A$. Fix any point $b \in W$. By Lemma 2.3, there exists $h \in C(G, Y)$ such that $h(a)=b$ and $h(x)=g(x)$ for all $x \in K$, which proves the theorem.

Next, we consider compositions of our operators with other suitable operators. But, before this, observe that if $T$ and $S$ are operators on $C(G, Y)$, then

$$
R(T S, A, B)=S^{-1}(R(T, A, B)) \quad(A \subset X, B \subset Y) .
$$

Theorem 3.2. Assume that $T, S$ are operators on $C(G, Y)$, in such a way that $T$ is DI (respectively, omnipresent) and $S$ is linear and onto. Then TS is DI (respectively, omnipresent). 
Proof. The Open Mapping Theorem (recall that $C(G, Y)$ is a Fréchet space) tells us that $S$ takes open sets into open sets, hence $S^{-1}(R(T, A, B))$ is dense whenever $R(T, A, B)$ is. Now, equality (3.1) and the definitions of DI and omnipresent operators prove the assertion.

Corollary 3.3. If $S$ is an onto linear operator on $C(G, Y)$, then $S$ is a DI (hence omnipresent) operator.

Proof. Combine Theorems 3.1 and 3.2 .

Our next result establishes that if a DI (or omnipresent) operator is perturbed by an operator which is 'controlled' near the boundary, then a new 'wild' operator is obtained, at least when $G$ is relatively compact in $X$. This happens, for instance, when $X$ itself is compact.

Theorem 3.4. Suppose that $\bar{G}$ is compact. Let $T$ be a DI (respectively, omnipresent) operator on $C(G, Y)$. Assume that $S$ is an operator on $C(G, Y)$ satisfying that, for every $f \in C(G, Y)$ and every $t \in \partial G$, there exists $\lim _{x \rightarrow t} S f(x) \in Y$. Then $T+S$ is a $D I$ (respectively, omnipresent) operator.

Proof. Assume that $T$ is a DI-operator. Fix a non-relatively compact subset $A \subset G$, a non-empty open subset $W \subset Y$ and a basic neighbourhood $D(g, K, \varepsilon)$ as in (2.1). In order that $T+S$ be a DI-operator, it must be proved that $R(T+S, A, W)$ is dense.

There exist a vector $w \in Y$ and a neighbourhood $U$ of the origin in $Y$ with $W \supset$ $w+U+U$. Since $\bar{G}$ is compact and $A$ is not relatively compact in $G, \bar{A}$ must be a compact set which is not included in $G$, so there exists $t \in \bar{A} \backslash G$, whence $t \in \bar{A} \cap \partial G$. But there exists $y:=\lim _{x \rightarrow t} S f(x) \in Y$. We can then find an open subset $V \subset X$ containing $t$ such that $(S f)(x) \in y+U$ whenever $x \in V$. Clearly, $t$ is also in the closure of $A \cap V$, hence $A \cap V$ is not relatively compact in $G$ because $t \notin G$. By hypothesis, the set $R(T, A \cap V, w-y+U)$ is dense in $C(G, Y)$. But if $f \in R(T, A \cap V, w-y+U)$, then there exists $a \in A \cap V$ with $T f(a) \in w-y+U$. Therefore,

$$
(T+S) f(a) \in w-y+U+y+U=w+U+U \subset W,
$$

that is, $f \in R(T+S, A, W)$. Thus,

$$
R(T+S, A, W) \supset R(T, A \cap V, w-y+U),
$$

which proves the density of $R(T+S, A, W)$. In the case that $T$ is omnipresent, the proof is analogous (but easier) and it is left to the reader.

We now turn our attention to the question of whether the existence of one 'wild' function under the action of $T$ implies that $T$ itself is 'wild'. Pointwise stability (see $\S 2$ ) makes its first appearance. 
Theorem 3.5. Let $T$ be an operator on $C(G, Y)$. We have the following.

(a) If $R(T, A, W) \neq \emptyset$ for every non-relatively compact subset $A \subset G$ and every nonempty open subset $W \subset Y$ and $T$ is pointwise stable near the boundary, then $T$ is a DI-operator.

(b) If $M(T, A) \neq \emptyset$ for every non-relatively compact subset $A \subset G$ and $T$ is pointwise stable near the boundary, then $T$ is a DI-operator.

(c) If $R(T, V, W) \neq \emptyset$ for every $V \in O(\partial G)$ and every non-empty open subset $W \subset Y$ and $T$ is somewhere pointwise stable near the boundary, then $T$ is omnipresent.

(d) If $C h(T) \neq \emptyset$ and $T$ is somewhere pointwise stable near the boundary, then $T$ is omnipresent.

Proof. Statements (b) and (d) follow, respectively, from (a) and (c), and from (2.2) and (2.3).

Let us prove (a). It must be shown that each set $R(T, A, W)$ is dense. Fix a basic neighbourhood $D(g, K, \varepsilon)$ as in (2.1). Clearly, $A \backslash M$ is not relatively compact in $G$, where $M$ is the compact subset appearing in the definition of pointwise local stability. By hypothesis, $R(T, A \backslash M, W)$ is not empty; pick $f$ in this set. Because $T$ is pointwise stable near the boundary, there is a point $b \in G \backslash K$ such that $T h(a) \in W$ whenever $h(b)=f(b)$. By Lemma 2.3, we may arrange for $h$ to agree with $g$ on $K$. Such an $h$ is in $R(T, A, W) \cap D(g, K, \varepsilon)$, which yields the desired density property. The proof of part (c) is analogous: use the fact that $R(T, V \backslash M, W)$ is not empty, where $V \in O(\partial G), M$ is as above and $W$ is a non-empty open subset of $Y$. The details are left to the reader.

In the following theorem, another condition that yields wild behaviour for $T$ starting from the wild behaviour of a single function is stated, this time with the help of linearity.

Theorem 3.6. Suppose that $T$ is a linear operator on $C(G, Y)$. We have the following.

(a) If $M(T, A) \neq \emptyset$ for every non-relatively compact subset $A \subset G, \bar{G}$ is compact and there exists a dense subset $\mathcal{D}$ such that for every $t \in \partial G$ and every $g \in \mathcal{D}$ there is an open subset $V \subset X$ containing $t$ such that $T g(V \cap G)$ is relatively compact in $Y$, then $T$ is $D I$.

(b) If $C h(T) \neq \emptyset$ and there exists a dense subset $\mathcal{D}$ such that for every $t \in \partial G$ and every $g \in \mathcal{D}$ the limit $\lim _{x \rightarrow t} T g(x)$ exists in $Y$, then $T$ is omnipresent.

Proof. Starting from (a), fix a non-relatively compact subset $A \subset G$. Then there is a sequence $\left(x_{n}\right) \subset A$ with $x_{n} \rightarrow t(n \rightarrow \infty)$ for some $t \in \partial G$ due to the compactness of $\bar{G}$. For fixed $g \in \mathcal{D}$ we may assume, by taking a subsequence if necessary, that $T g\left(x_{n}\right) \rightarrow y$ $(n \rightarrow \infty)$ for some vector $y \in Y$. This can be accomplished by using the hypothesis of relative compactness of $T g(V \cap G)$. But $\left(x_{n}\right)$ is non-relatively compact in $G$, so there 
exists $f \in M\left(T,\left(x_{n}\right)\right)$, hence $\overline{\left\{T f\left(x_{n}\right): n \in \mathbb{N}\right\}}=Y$. Consequently,

$$
\begin{aligned}
\overline{T(f+g)(A)} & \supset \overline{\left\{T(f+g)\left(x_{n}\right): n \in \mathbb{N}\right\}} \\
& =\overline{\left\{T f\left(x_{n}\right)+T g\left(x_{n}\right): n \in \mathbb{N}\right\}} \\
& =Y+y \\
& =Y,
\end{aligned}
$$

therefore $f+\mathcal{D} \subset M(T, A)$, whence $M(T, A)$ is dense and $T$ is DI by Proposition 2.2. The proof of (b) is analogous, and left to the reader.

Observe that the boundary condition imposed in (a) is weaker than that imposed in (b).

Following [6], we give an example showing that the existence of a single boundarychaotic function does not imply omnipresence. Consider the case $X=\mathbb{R}=Y, G=$ $(-1,1)$. Fix a function $\psi \in C h(I)$, where $I$ is the identity operator. If $T$ is the operator given by

$$
T f(x)=\psi\left(\frac{x}{1+|f(0)|}\right) \quad(x \in(-1,1)),
$$

then $C h(T)=\{f \in C((-1,1), \mathbb{R}): f(0)=0\}$, so $C h(T)$ is non-empty but it is of first category. Hence $T$ is not omnipresent, so it is not DI.

On the contrary, we do not know whether an operator $T$ is DI under the unique condition that $M(T, A) \neq \emptyset$ for all non-relatively compact subsets $A \subset G$. Observe that the operator given above is not linear, which opens the following question, the answer to which we do not know to date.

\section{If $T$ is linear and $C h(T) \neq \emptyset$, is $T$ omnipresent?}

Next we study the relationship between 'wild' behaviour of an operator and the 'size' of its range. Consider again the case $X=\mathbb{R}=Y, G=(-1,1)$. Then the operator $T$ given by

$$
T f(x)=f\left(\frac{1}{2} x\right)
$$

has dense range (because all polynomials are in the range of $T$ ) but $T$ is not omnipresent (so it is not DI); in fact, $C h(T)=\emptyset$ because each $T f$ has continuous extension to $[-1,1]$. Conversely, the operator $P$ given by

$$
P f(x)=f(x)-f(0)
$$

is DI, hence omnipresent (apply Theorem 3.1 together with Theorem 3.4 with $T=I$ and $S$ given by $S f(x)=-f(0)$ ). Nevertheless, $P$ does not have dense range because $P f$ vanishes at 0 for every $f$. But at least we are able to show that the pointwise density of the range together with pointwise stability causes wild behaviour (see Theorem 3.7). Note the intertwining between these diverse properties. 
Theorem 3.7. Assume that $T$ is an operator on $C(G, Y)$.

(a) If $T$ is pointwise stable near the boundary and has pointwise dense range near the boundary, then $T$ is a DI-operator.

(b) If $T$ is pointwise stable near the boundary and has somewhere pointwise dense range near the boundary, then $T$ is omnipresent.

(c) If $T$ is somewhere pointwise stable near the boundary and has pointwise dense range near the boundary, then $T$ is omnipresent.

Proof. Assume that the hypotheses of (a) are fulfilled. Fix a set $R(T, A, W)$ and also a basic neighbourhood $D(g, K, \varepsilon)$ as in (2.1). There is a compact subset $M \subset G$ such that the set $\{T f(x): f \in C(G, Y)\}$ is dense in $Y$ for every $x \in G \backslash M$ and, in addition, $M$ satisfies the condition of stability with respect to $K$ (see $\S 2$ ). Take an open neighbourhood $U$ of the origin in $Y$ and a vector $w \in Y$ with $w+U+U \subset W$. Since $A \backslash M$ is not empty, we can pick a point $a$ in it. Then $\{T f(a): f \in C(G, Y)\}$ is dense in $Y$, therefore a continuous function $f$ can be found in order that $T f(a) \in w+U$ holds. Furthermore, there is $b \in G \backslash K$ satisfying the condition of stability.

An application of Lemma 2.3 provides us with a continuous function $h$ such that $h=g$ on $K$ and $h(b)=f(b)$. Then $h \in D(g, K, \varepsilon)$ and

$$
T h(a) \in T f(a)+U \in w+U+U \subset W,
$$

so $h \in R(T, A, W)$, which completes the proof of (a). The proofs of (b) and (c) are analogous, so they are omitted.

Of course, condition (a) is stronger than (b) and (c). A closer look at the last proof reveals that the density condition in (a) can be replaced by the following: for each nonrelatively compact subset $A \subset G$, the set $\{T f(a): a \in A, f \in C(G, Y)\}$ is dense in $Y$.

\section{Classical examples: composition and multiplication operators}

Our attention is now focused on characterizing the DI property and the omnipresence of several concrete examples of operators, namely, the multiplication operator $M_{\psi}$, the right composition operator $C_{\varphi}$, and the left composition operator $L_{\alpha}$. Let us recall the definitions. We denote by $\mathbb{K}$ the scalar field of $Y$. We assume from now on that $\psi: G \rightarrow \mathbb{K}$, $\varphi: G \rightarrow G$ and $\alpha: Y \rightarrow Y$ are continuous mappings. Then $M_{\psi}, C_{\varphi}, L_{\alpha}$ are, respectively, defined as

$$
M_{\psi} f(x)=\psi(x) \cdot f(x), \quad C_{\varphi} f(x)=f(\varphi(x)) \quad \text { and } \quad L_{\alpha} f(x)=\alpha(f(x)),
$$

for $x \in G$ and $f \in C(G, Y)$. Observe that $M_{\psi}$ and $C_{\varphi}$ are linear. The promised characterizations will be made in the following three theorems. 


\section{Theorem 4.1.}

(A) The following properties are equivalent.

(a) The operator $M_{\psi}$ is $D I$.

(b) The set $M\left(M_{\psi}, A\right) \neq \emptyset$ is non-empty for every non-relatively compact subset $A$ of $G$.

(c) The zero set of $\psi$, i.e. the set $\mathcal{Z}(\psi)=\{x \in G: \psi(x)=0\}$, is relatively compact in $G$.

(B) The following properties are equivalent.

(a) The operator $M_{\psi}$ is omnipresent.

(b) The set $C h\left(M_{\psi}\right) \neq \emptyset$.

(c) For each $V \in O(\partial G)$ there is $a \in V \cap G$ with $\psi(a) \neq 0$.

Proof. From Proposition 2.2, it is trivial that (a) implies (b) in both (A) and (B). Suppose that (b) holds in (A). By way of contradiction, if $\mathcal{Z}(\psi)$ were not relatively compact in $G$, the set $M\left(M_{\psi}, \mathcal{Z}(\psi)\right)$ would have at least one member $f$, which is absurd because $M_{\psi} f(\mathcal{Z}(\psi))=\{0\}$. Hence (b) implies (c) in (A).

The same holds for (B). Indeed, assume that there is $f \in C h\left(M_{\psi}\right)$ and that, again by way of contradiction, there exists a set $V \in O(\partial G)$ such that $\psi=0$ on $V \cap G$. If $W$ is any non-empty open subset of $Y$ not containing the origin, we have $f \in R\left(M_{\psi}, V, W\right)$ but $M_{\psi} f(a)=\psi(a) \cdot f(a)=0 \notin W$ for all $a \in V \cap G$, which is a contradiction.

On the other hand, it is evident that $M_{\psi}$ is always pointwise stable near the boundary (take $M=K$ and $b=a$ in the definition of stability) and, under (c), $M_{\psi}$ has either pointwise dense range near the boundary (for $(\mathrm{A})$ ) or somewhere pointwise dense range near the boundary (for (B)). Then Theorem 3.7 comes to our aid to show (a), and the proof is finished.

The last result furnishes an example of an omnipresent, linear operator that is not a DI-operator. Indeed, take $X=\mathbb{R}=Y, G=(0,1)$ and $\psi(x)=\sin (1 / x)$. The desired operator is $M_{\psi}$.

Remark 4.2. It is not difficult to establish the following assertions combining $M_{\psi}$ with an operator $T$ on $C(G, Y)$.

(i) If $T$ satisfies the hypothesis of Theorem 3.7 (a) and $\mathcal{Z}(\psi)$ is not relatively compact in $G$, then $M_{\psi} T$ satisfies the hypothesis of Theorem 3.7 (a), so it is DI.

(ii) If $T$ satisfies the hypothesis of Theorem 3.7 (b) (respectively, (c)) and $\mathcal{Z}(\psi)$ is not relatively compact in $G$, then $M_{\psi} T$ satisfies the hypothesis of Theorem 3.7 (b) (respectively, (c)), so it is omnipresent.

(iii) If $T$ satisfies the hypothesis of Theorem 3.7 (a) and for every $V \in O(\partial G$ ) there is $a \in V \cap G$ with $\psi(a) \neq 0$, then $M_{\psi} T$ satisfies the hypothesis of Theorem 3.7 (b), so it is omnipresent. 
By Corollary 3.3, the operator $C_{\varphi}$ is DI whenever $\varphi$ is a homeomorphism. However, as promised, we get sharp conditions. Recall that a continuous mapping $\varphi: G \rightarrow G$ is said to be proper whenever the preimage $\varphi^{-1}(K)$ of each compact subset $K \subset G$ is also compact. It is easy to see that $\varphi$ is proper if and only if $\varphi(A)$ is non-relatively compact in $G$ for every non-relatively compact subset $A \subset G$.

\section{Theorem 4.3.}

(A) The following properties are equivalent.

(a) The operator $C_{\varphi}$ is DI.

(b) The set $M\left(C_{\varphi}, A\right)$ is non-empty for every non-relatively compact subset $A \subset$ $G$.

(c) The mapping $\varphi$ is proper.

(B) The following properties are equivalent.

(a) The operator $C_{\varphi}$ is omnipresent.

(b) The set $C h\left(C_{\varphi}\right) \neq \emptyset$.

(c) The set $\varphi(V \cap G)$ is non-relatively compact in $G$ for every $V \in O(\partial G)$.

Proof. We will make use of the following identities. For every operator $T$ on $C(G, Y)$, every $A \subset G$ and every $B \subset Y$, we have

$$
R\left(C_{\varphi} T, A, B\right)=R(T, \varphi(A), B)
$$

and, from (2.3) and (4.1),

$$
M\left(C_{\varphi} T, A\right)=M(T, \varphi(A)) .
$$

Firstly, we have again by Proposition 2.2 that (a) implies (b) in both (A) and (B). Assume that (b) holds in (A) and fix a non-relatively compact subset $A \subset G$. Then there is $f \in M\left(C_{\varphi}, A\right)=M(I, \varphi(A))$, where the last equality is due to (4.2) as applied on the identity operator. Therefore, $f(\varphi(A))$ is dense in $Y$, which forces $\varphi(A)$ to be non-relatively compact in $G$, which gives (c).

If (b) now holds in (B), then we can pick $f \in C h\left(C_{\varphi}\right)$. Assuming $V \in O(\partial G)$, the set $C_{\varphi} f(V \cap G)=f(\varphi(V \cap G))$ should be dense in $Y$, hence $\varphi(V \cap G)$ cannot be relatively compact in $G$, and this is (c) of (B).

Again by (4.2), we get $M\left(C_{\varphi}, A\right)=M(I, \varphi(A))$. Then (c) implies (a) in (A): just combine Proposition 2.2(a) and Theorem 3.1. Finally, starting from (c) of (B), in order to prove (a) we should show that $R\left(C_{\varphi}, V, W\right)=R\left(C_{\varphi}, V \cap G, W\right)$ is dense in $C(G, Y)$ for every $V \in O(\partial G)$ and every non-empty open subset $W \subset Y$. But, by (4.1), $R\left(C_{\varphi}, V \cap G, W\right)=R(I, \varphi(V \cap G), W)$, and this set is dense because $I$ is DI (not only omnipresent!). 
Remark 4.4. A systematic application of (4.1) allows us to arrive at the following statements.

(i) The operator $C_{\varphi} T$ is DI for each DI-operator $T$ if and only if $\varphi$ is proper.

(ii) The operator $C_{\varphi} T$ is omnipresent for each DI-operator $T$ if and only if $\varphi(V \cap G)$ is non-relatively compact in $G$ for all $V \in O(\partial G)$.

(iii) The operator $C_{\varphi} T$ is omnipresent for each omnipresent operator $T$ if $\varphi$ is 'open in the boundary', that is, given $V \in O(\partial G)$ there exists $V^{\star} \in O(\partial G)$ with $V^{\star} \cap G \subset$ $\varphi(V \cap G)$.

Observe that a new example of a linear, omnipresent, non-DI-operator may be extracted from Theorem 4.3. Indeed, $X=[0,1], G=[0,1)$ and $Y=\mathbb{R}$. If $\varphi(x)=$ $x|\sin (1 /(1-x))|$, then $C_{\varphi}$ gives a suitable operator. Nevertheless, it is not possible to construct a similar example by employing left composition operators, as we can see in the next surprising (and final) theorem.

Theorem 4.5. The next seven properties are equivalent.

(a) The operator $L_{\alpha}$ is DI.

(b) The operator $L_{\alpha}$ is omnipresent.

(c) The set $M\left(L_{\alpha}, A\right)$ is non-empty for every non-relatively compact subset $A \subset G$.

(d) The set $C h\left(L_{\alpha}\right)$ is non-empty.

(e) The operator $L_{\alpha} T$ is DI for every DI-operator $T$.

(f) The operator $L_{\alpha} T$ is omnipresent for every omnipresent operator $T$.

(g) The function $\alpha$ has dense range, i.e. $\overline{\alpha(Y)}=Y$.

Proof. The proof can be accomplished by the interested reader if the following facts are applied: the identity is a DI-operator; every DI-operator is omnipresent; the characterization given in Proposition 2.2; $\alpha$ has dense range if and only if $\alpha^{-1}(W)$ is non-empty for each non-empty open subset $W \subset Y$; and for every operator $T$ on $C(G, Y)$, every $A \subset G$ and every $B \subset Y, R\left(L_{\alpha} T, A, B\right)=R\left(T, A, \alpha^{-1}(B)\right)$.

The equivalence of $(\mathrm{a})-(\mathrm{g})$ can now be proved in the following order:

$$
\begin{aligned}
& (\mathrm{a}) \Rightarrow(\mathrm{b}) \Rightarrow(\mathrm{d}) \Rightarrow(\mathrm{g}) \Rightarrow(\mathrm{e}) \Rightarrow(\mathrm{a}), \\
& (\mathrm{a}) \Rightarrow(\mathrm{c}) \Rightarrow(\mathrm{g}) \Rightarrow(\mathrm{f}) \Rightarrow(\mathrm{b}) .
\end{aligned}
$$

As a final comment, we point out that $L_{\alpha}$ is, clearly, pointwise stable near the boundary, which yields at once the equivalences (a) $\Leftrightarrow(\mathrm{c})$ and (b) $\Leftrightarrow(\mathrm{d})$ (cf. Theorem 3.5). Thus, an alternative chain of implications is available for the proof of Theorem 4.5.

Acknowledgements. The authors have been partly supported by Plan Andaluz de Investigación de la Junta de Andalucía. 


\section{References}

1. L. BERnAL-GONZÁLEZ, Omnipresent holomorphic operators and maximal cluster sets, Colloq. Math. 63 (1992), 315-322.

2. L. Bernal-GonzÁlez, Plane sets having dense holomorphic images, Rev. Roumaine Math. Pures Appl. 40 (1995), 567-569.

3. L. Bernal-GonzÁlez And M. C. Calderón-Moreno, Holomorphic T-monsters and strongly omnipresent operators, J. Approx. Theory 104 (2000), 204-219.

4. L. Bernal-GonzÁlez And M. C. CAlderón-Moreno, Operators with dense images everywhere, J. Math. Analysis Applic. 263 (2001), 95-109.

5. L. Bernal-González, M. C. Calderón-Moreno and K. G. Grosse-Erdmann, Strongly omnipresent integral operators, Integ. Eqns Operat. Theory 44 (2002), 397-409.

6. L. Bernal-González, M. C. Calderón-Moreno and K. G. Grosse-Erdmann, Strongly omnipresent operators: general conditions and applications to composition operators, J. Austral. Math. Soc. A 72 (2002), 335-348.

7. M. C. CALderón-Moreno, Holomorphic operators and plane sets with dense images, Complex Variables 47 (2002), 167-176.

8. E. F. Collingwood And A. J. Lohwater, The theory of cluster sets (Cambridge University Press, 1966).

9. J. B. ConWAy, Functions of one complex variable (Springer, 1986).

10. K. G. Grosse-Erdmann, Holomorphe Monster und universelle Funktionen, Mitt. Math. Sem. Giessen 176 (1987).

11. K. G. Grosse-Erdmann, Universal families and hypercyclic operators, Bull. Am. Math. Soc. 36 (1999), 345-381.

12. D. Hinrichsen AND J. L. Fernández, Topología general (Urmo, Bilbao, 1977).

13. J. HoRváth, Topological vector spaces and distributions, vol. 1 (Addison-Wesley, 1966).

14. W. LuH, Holomorphic monsters, J. Approx. Theory 53 (1988), 128-144.

15. W. LuH, Multiply universal holomorphic functions, J. Approx. Theory 89 (1997), 135155.

16. W. Luh, V. A. Martirosian And J. Müller, T-universal functions with lacunary power series, Acta Sci. Math. 64 (1998), 67-79.

17. J. Margalef, E. Outerelo And J. L. Pinilla, Topología, vol. 2, p. 231 (Alhambra, Madrid, 1979).

18. J. Margalef, E. Outerelo and J. L. Pinilla, Topología, vol. 3, pp. 10-19 (Alhambra, Madrid, 1980).

19. K. Noshiro, Cluster sets (Springer, 1960).

20. I. SCHNEIDER, Schlichte Funktionen mit universellen Approximationseigenschaften, Mitt. Math. Sem. Giessen 230 (1997), 1-72. 